\title{
An efficient four points modified explicit group poisson solver
}

\begin{abstract}
In this paper, we introduce a four points modified explicit group method for solving a two dimensional Poisson equation with Dirichlet boundary condition. The method is shown to be superior compared to the existing four pointsï explicit group and explicit decoupled group methods due to D. J. Evans and M. J. Biggins (1982) and A. R. Abdullah (1991), respectively, Some experiment results of the test problem are given in order to confirm our claim.
\end{abstract}

Keyword: Poisson equation; Modified Explicit Group (MEG); Explicit Group (EG); Explicit Decoupled Group (EDG); Performance evaluation 